\title{
Assessment of the energy balance gap for ship-FOWT collision simulations with LS-DYNA/MCOL
}

\author{
Í. Ladeira \& H. Le Sourne \\ ICAM, Nantes, France
}

S. Echeverry \& P. Rigo

Université de Liège, Liège, Belgium

\begin{abstract}
Non-linear finite element (NLFE) codes are a consolidated tool in the crashworthiness analysis of ships and offshore structures, employed in the design of fixed offshore wind turbines (OWT) and, more recently, for floating offshore wind turbines (FOWT). In this latter case, additional complexities associated to large rigid body rotations and strong hydrodynamic effects require the use of algorithms capable of coupling external dynamics and internal mechanics. One of the available methodologies uses the NLFE package LSDyna combined with the rigid body dynamics program MCOL. This approach has been previously validated for ship-ship collisions. However, some inconsistencies arise for ship-FOWT impacts, particularly in the global energy balance. A parcel of the total energy input is seemingly lost, contradicting the expected energy conservancy. Therefore, the objective of this study is to understand the issue by performing LS-Dyna/MCOL simulations of ship-FOWT collisions, where main influencing factors can be observed independently. Simplified numerical models are developed for this purpose. The study provides a detailed understanding of the issue and its source.
\end{abstract}

\section{INTRODUCTION}

\subsection{Context}

In the scope of the ColFOWT (Collision against Floating Offshore Wind Turbines) project currently undergoing in France, a simplified analytical simulation tool for the analysis of ship-FOWT collisions is under development. Thanks to its rapidity compared to nonlinear finite element simulations, such a tool will allow design offices to assess the consequences of a collision in terms of ship and wind turbine damage, considering all the scenarios requested for a complete risk analysis. Developments are to be validated against results obtained with the non-linear explicit FEM code LS-Dyna coupled with the rigid body dynamics program MCOL. This semi-coupled approach has been consolidated for ship-ship collisions (Le Sourne et al. 2001 and Le Sourne et al. 2003) and is expected to be reliable for ship-FOWT collisions even though, in this particular case, strong hydrodynamics effects combined with large surge and pitch motions of the floating platform add complexity to the problem.

LS-Dyna/MCOL simulations of a spar platform FOWT subjected to ship impact have been carried out by Echeverry et al. (2020). Although results seem to be reasonably physical for preliminary validations, these numerical simulations are not yet fully reliable, particularly when it comes to the global energy balance of the system. It seems that a parcel of the initial kinetic energy of the striking ship is lost when using LS-Dyna in conjunction with MCOL. Moreover, as confirmed recently by Rudan et al. (2019), the rigid body movement of the struck floater becomes unrealistic after a certain period of time.

Therefore, the objective of this study is to assess the reliability of the LS-Dyna/MCOL package for this application, providing a detailed explanation for the inconsistency of some results and guidelines for LSDyna/MCOL simulations. This is achieved with the use of ship-FOWT collision simplified models where governing parameters can be isolated and their individual influence more clearly observed. Details of the simplified models considered in the present study are given in Section 3.

\subsection{Ship Collision Analysis}

Ship collisions are complex events that involve different types of physical interactions and energy transfer mechanisms, typically divided into two branches: the external dynamics and the internal mechanics, as initially suggested by Minorsky (1958).

The external dynamics deals with the global motions of striking and struck vessels, before, during and after collision. It predicts how much of the initial ki- 
netic energy is dissipated into deformation mechanisms, residual motion or hydrodynamic effects. The internal mechanics, on the other hand, describes the different deformation mechanisms such as local crushing, global bending and elastic vibrations that contribute to dissipate the energy. The approach consisting in solving successively external dynamics and internal mechanics has been widely applied in ship collision and grounding analyses for its simplicity and reasonable accuracy in predicting the deformation of the involved structures. However, results for the trajectory of the colliding bodies and resultant structural responses are not as accurate and can even be underestimated in some collision scenarios, as shown by Le Sourne et al. (2001), Brown (2002) and Tabri (2012). Therefore, alternative approaches using semi or fullycoupled algorithms have been developed in the 20 past years.

In a semi-coupled approach, external dynamics and internal mechanics are solved in parallel with separate routines that are mutually and iteratively updated. Following this approach, Le Sourne et al. (2001) for instance use LS-Dyna to calculate the crushing force acting on a submarine impacted by a surface ship. The force components and resulting moments at the vessel's center of gravity $(\mathrm{CoG})$ are then transmitted to the rigid body dynamics program MCOL, which solves the 6-DOF equation of motion for each vessel. MCOL uses a potential flow theory to calculate the hydrodynamic forces and moments due to the surrounding fluid (Le Sourne 2007). Hydrodynamic characteristics of the vessels such as water added mass, wave radiation damping, and hydrostatic restoring matrices must be calculated preliminarily using a sea-keeping code. Further examples of LS-Dyna/MCOL simulations may be found in Le Sourne et al. (2003), Rudan et al. (2019) and Paboeuf et al. (2015).

The semi-coupled approach is also applied by $\mathrm{Yu}$ \& Amdahl (2016), Yu et al. (2016a) and Yu et al. (2016b), where the contact force is as well extracted from LS-Dyna simulation. However, a 6-DOF maneuvering model based on a series of non-dimensional experimental coefficients is used to calculate the hydrodynamic loads, which are applied to the CoG of the colliding structures with a user-defined load subroutine available in LS-Dyna.

In both approaches, the fluid-structure interaction is computed using an external hydrodynamic solver. Alternatively, in a fully coupled model, the fluid is explicitly represented with the use of either Eulerian or Arbitrary Eulerian Lagrangian (ALE) grids coupled with a Lagrangian mesh for the structures. This fully coupled approach has been tested for ship collisions in Rudan et al. (2019), Lee et al. (2012) and Song et al. (2017). Nevertheless, it is generally concluded that gains in accuracy do not outweigh the required modelling and computational efforts for this technique, therefore limiting its industrial application at present.

\section{PROBLEM DESCRIPTION}

In a ship-FOWT collision event, the total initial kinetic energy is transferred into deformation and sliding energies, residual post-impact kinetic energy and work performed by the hydrodynamic forces. Other energy dissipation mechanisms may also occur but are generally negligible.

The energy balance of the system is thus given by the following equation:

$$
K_{0}=U_{\text {ship }}+U_{\text {fowt }}+K_{\text {ship }}+K_{\text {fowt }}+E_{\text {hydro }}
$$

where $K$ denotes kinetic energy, $U$ refers to deformation energy and $E_{\text {hydro }}$ is the energy related to hydrodynamic forces. This latter energy can be decomposed into work performed by hydrostatic restoring forces $H$, viscous drag forces $V$ and wave damping forces $W$. If the FOWT is at rest when the collision occurs, the sum of all above energies equals the initial striking ship's kinetic energy $K_{0}$.

Using the LS-Dyna/MCOL semi-coupled approach, the deformation energies $U_{\text {ship }}, U_{\text {fowt }}$ and $U_{\text {moor }}$ are computed by the finite element solver, while the kinetic energies $K_{\text {ship }}$ and $K_{\text {fowt }}$, as well as $E_{\text {hydro }}$, are post-processed from MCOL calculations.

Preliminary simulations using this approach have shown an inconsistency in the final energy balance, where the sum of all the energies does not remain equal to the initial kinetic energy $K_{0}$. In other words, some energy seems to be lost or incorrectly calculated when the LS-Dyna solver is used in conjunction with MCOL's subroutine.

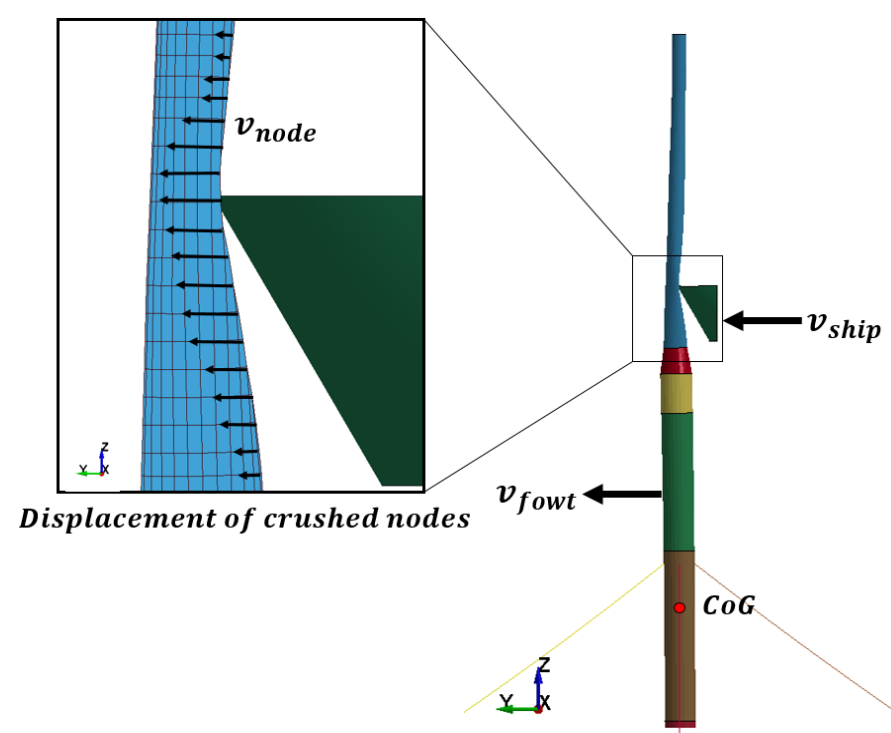

Figure 1. Relative movement of the FOWT's crushed nodes. 
One hypothesis that explains this issue is based on the fact that MCOL calculates the FOWT's kinetic energy $K_{\text {fow } t}$ with respect to its CoG, taking into account the inertia forces related to the water added mass. $K_{\text {fowt }}$ is thus related to the rigid body motion of the structure. However, the reality is that part of the FOWT's kinetic energy comes from the nodes located in the impacted area of the structure, which are moved with a given velocity $v_{\text {node }}$ (Figure 1). This so-called "crushed nodes kinetic energy" is actually not considered by MCOL and, as discussed later, could be significant, therefore originating the gap in the final energy balance.

\section{ASSESSMENT}

Simplified ship-FOWT collision models are set up to test the influencing parameters that could be originating the issue with the LS-Dyna/MCOL simulation with better control.

First of all, gravity loads and mooring lines are disregarded, since they are most likely not related to the issue. Second, deformation is limited with the use of simplified geometries for both structures. Fully rigid and high stiffness deformable materials in addition to spring elements are also used for that purpose. The motion of the FOWT is limited or induced in specific ways with the use of constraints or by changing the impact location. This is done in order to mitigate the coupling of motion components.

Three simplified models are developed with the aforementioned general characteristics. These models are detailed and discussed in the following Sections 3.1, 3.2 and 3.3.

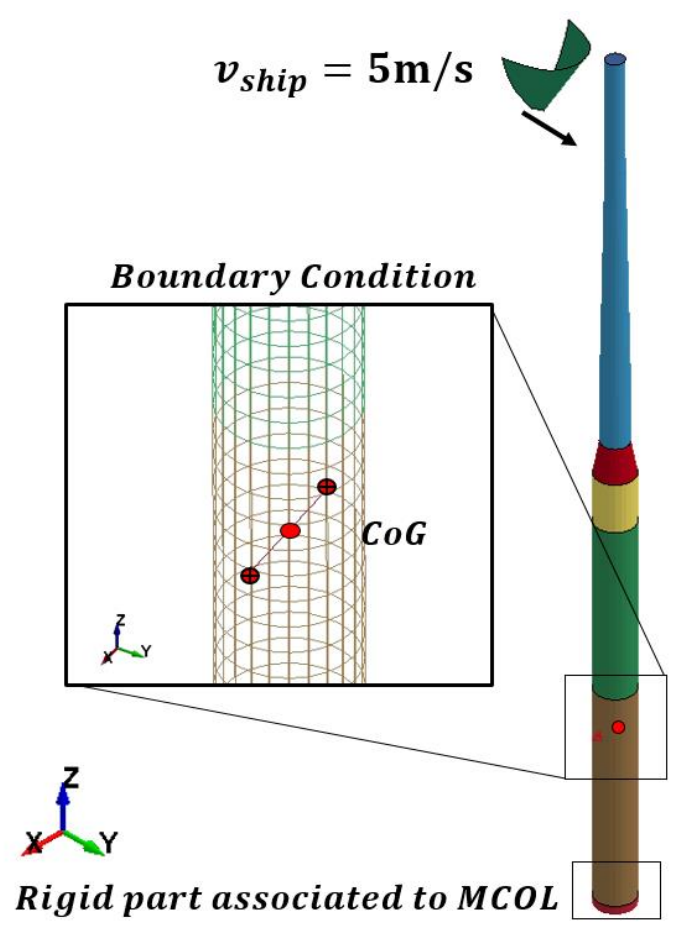

Figure 2. Model 1 setup. The applied boundary conditions are shown in the magnified detail.

\section{$3.1 \quad$ Model 1}

\subsubsection{Description}

Model 1, shown in Figure 2, is a simplification of the model used in Echeverry et al. (2020). Detailed dimensions can be found in this reference.

The simplified model is composed of a deformable FOWT and a rigid striking bow. The FOWT's bottom part (highlighted in the lower part of Figure ) is defined as rigid and associated to MCOL.

The ship impacts the FOWT at a high location relative to its CoG, traveling with a velocity $v_{\text {ship }}$ of $5 \mathrm{~m} / \mathrm{s}$. A constraint limiting the translations in all three directions and rotations around $y$ and $z$ axes is applied at the nodes adjacent to the FOWT's CoG, as highlighted in Figure. This configuration is intended to induce a large pitch movement in the FOWT, but completely restricting surge.

\subsubsection{Results and Discussion}

Figure 3 shows the time evolution of the energies during and after the impact up to 60 seconds. Curves for the internal energy $U$, residual kinetic energies $K$ and work of hydrostatic restoring forces $H$ are presented. The sum of these components Sum is also presented and can be compared to the ship initial kinetic energy $K_{0}$. The impact occurs at around $1.7 \mathrm{~s}$.

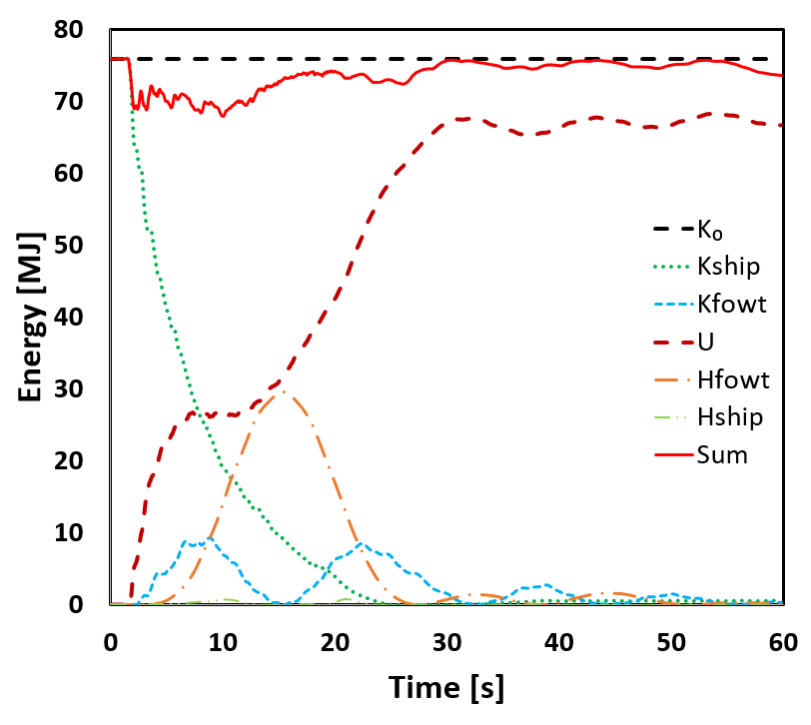

Figure 3. Energy balance - 60s.

The kinetic energy of the ship $K_{\text {ship }}$ decreases rapidly upon impact and the ship practically stops moving after 25 seconds. As expected, the impact induces an oscillatory pitch motion on the FOWT that is gradually dampened by the hydrodynamic damping forces, as demonstrated by $K_{\text {fowt }}$ and $H_{\text {fowt }}$ curves. The internal energy $U$ reaches a plateau (around 65MJ) when plastic deformations on the FOWT cease and only minor elastic vibrations remain.

The gap between initial kinetic energy $K_{0}$ (before impact) and the sum of all energies (during and after impact) is clearly observed. Note that the work performed by wave radiation and viscous drag damping 
forces is not plotted in the balance, as initially, it was thought that these components would be negligible. This matter is addressed in Section 3.2.

\subsection{Model 2}

\subsubsection{Description}

Model 2, shown in Figure 2, is a simplified version of Model 1 aiming to check the influence of the kinetic energy associated to the crushed nodes. To achieve this, the FOWT is represented by a rigid cylinder with similar dimensions and mass distribution. No constraints are applied to the FOWT, which is allowed to move freely.



Figure 2. Model 2 setup.

In order to prevent possible rigid-rigid contact instabilities between FOWT and impactor, the contacting part of the impactor is defined as a deformable semi-cylinder. Young's modulus and shell element thickness are intentionally increased with the objective of avoiding any plastic deformation. The semicylinder is connected by a spring to a rigid backing plate representing the rest of the striking ship. The spring's stiffness is experimentally tuned so that it compresses no more than the distance between the rigid and deformable parts, simulating the correspondent behavior of the original model and allowing some control over the resultant internal energy. Finally, the impactor is allowed to translate only along $y$ direction.

The FOWT's kinetic energy calculated by MCOL is expected to be correct, since no deformation occurs and the supposed kinetic energy of the crushed nodes is eliminated.

\subsubsection{Results and Discussion}

The striking semi-cylinder collides with the FOWT at approximately $1.7 \mathrm{~s}$. It is pushed backwards, compressing the spring until $5 \mathrm{~s}$, when the FOWT starts moving along the direction of the impact and the spring slowly decompresses. It returns to its initial length at around 8s. Figure 3 shows the energy balance for the 10s running time.

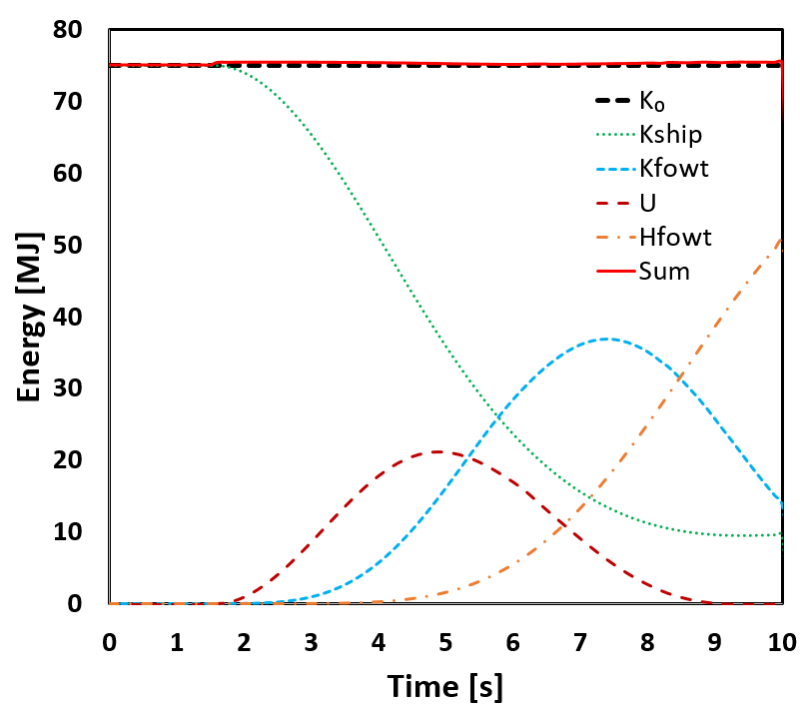

Figure 3. Energy balance - 10s.

During the contact phase, the striking ship decelerates and at the same time, the FOWT's angular acceleration increases up to $7.3 \mathrm{~s}$, subsequently dropping. Even though the FOWT's pitch motion begins as soon as the impact occurs, resulting hydrostatic restoring momentum becomes significant only 2 s later, countering the pitch movement of the floating structure.

The deformation energy of the spring $U$ increases from the beginning of the impact up to a peak corresponding to its maximum compression. In the absence of "crushed nodes" in the FOWT, the overall kinetic energy of the system, which is actually the sum of the FOWT's rigid body kinetic energy calculated by MCOL and the ship's kinetic energy calculated by LS-Dyna, is correct. There is no energy loss, as shown in Figure 3, where the total energy remains constant and equal to $K_{0}$. This demonstrates clearly that the energy loss detected in Figure comes from the relative velocity of the FOWT's crushed nodes (i.e. with respect to the $\mathrm{CoG}$ ) as this relative velocity is not accounted for by MCOL.

It is also worth to note that energies associated to wave and viscous damping forces have been found to be negligible in this simulation. However, as they might become significant in the long term, a second simulation is run up to 140 s. 


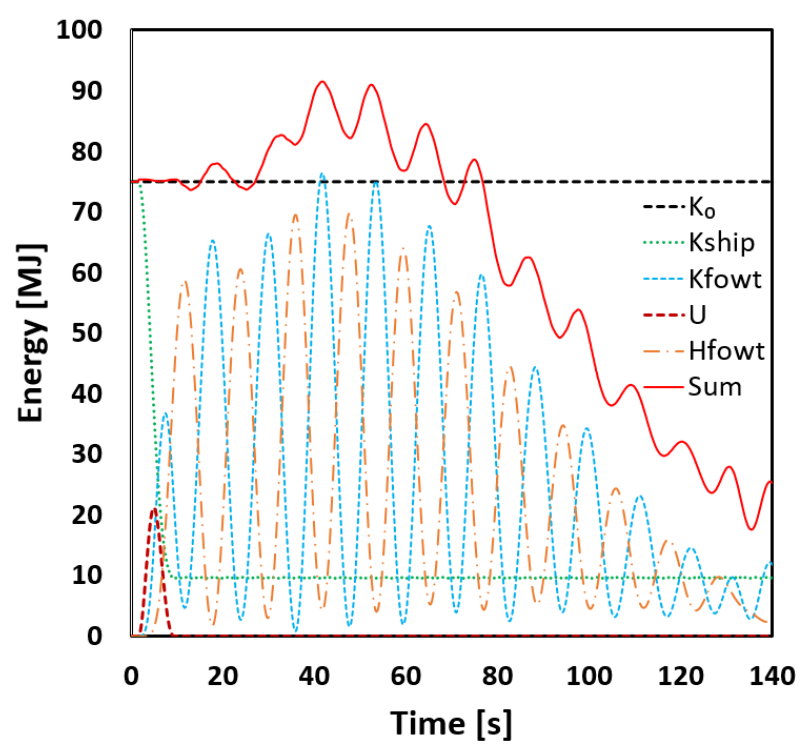

Figure 6. Energy balance - 140s.

In this simulation, the contact condition in LSDyna is intentionally limited to $10 \mathrm{~s}$, after which it ceases, and the impacting semi-cylinder is allowed to freely go through the FOWT. This avoids a secondary impact and facilitates the observation of the subsequent undisturbed pitch movement of the FOWT. Results are shown in Figure 6.

The total energy is approximately constant for the first 10 s of the simulation, as in the previous example. Nonetheless, afterwards, it exceeds unrealistically the initial kinetic energy $K_{0}$ and then sharply drops. It becomes evident that a significant amount of energy is missing.

By adding in Figure 7 the works of both wave $W$ and viscous $V$ damping forces, the total energy becomes constant all along the simulation, but the results are still unrealistic as both $W$ and $V$ exceed the initial energy of the system.

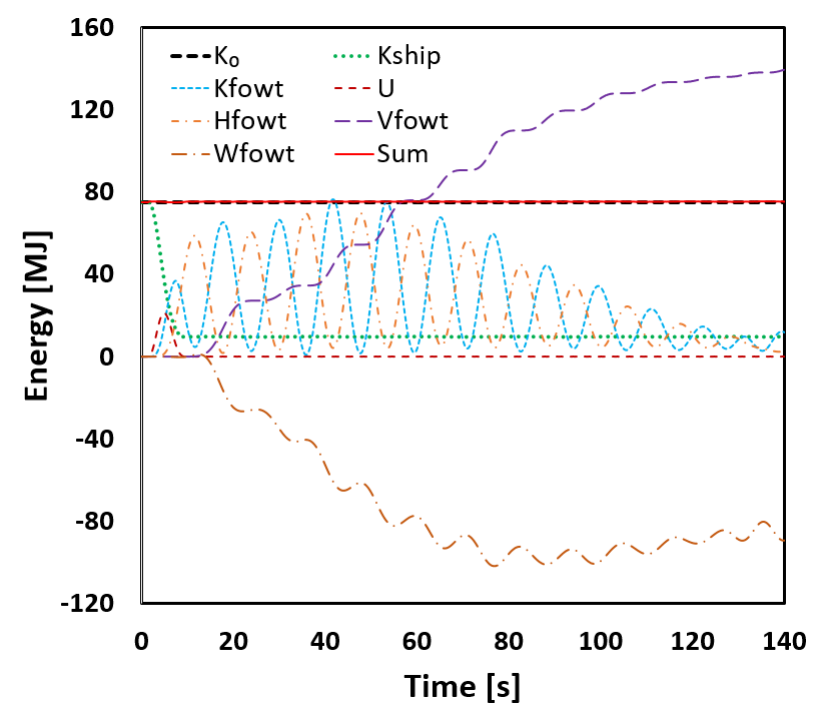

Figure 7. Energy balance - Wave and drag damping included $-140 s$.
In addition, the FOWT presents an incoherent physical movement, pitching in an unexpected way. This can be observed from the profiles of $K_{\text {fowt }}$ and $H_{\text {fowt }}$ : the pitch oscillations increase unrealistically after the end of the contact between the structures before decaying, when in reality the wave radiation should immediately damp the FOWT's pitch movement.

An assessment of the individual influences of wave and viscous drag damping is thus performed by comparing results obtained for the current model with three different configurations: in the first one, no damping is considered, while the second one includes only viscous damping and the third only wave radiation damping. Resulting energy balances are presented in Figures 8, 9 and 10, respectively.



Figure 8. Energy balance - No damping included - 140s.

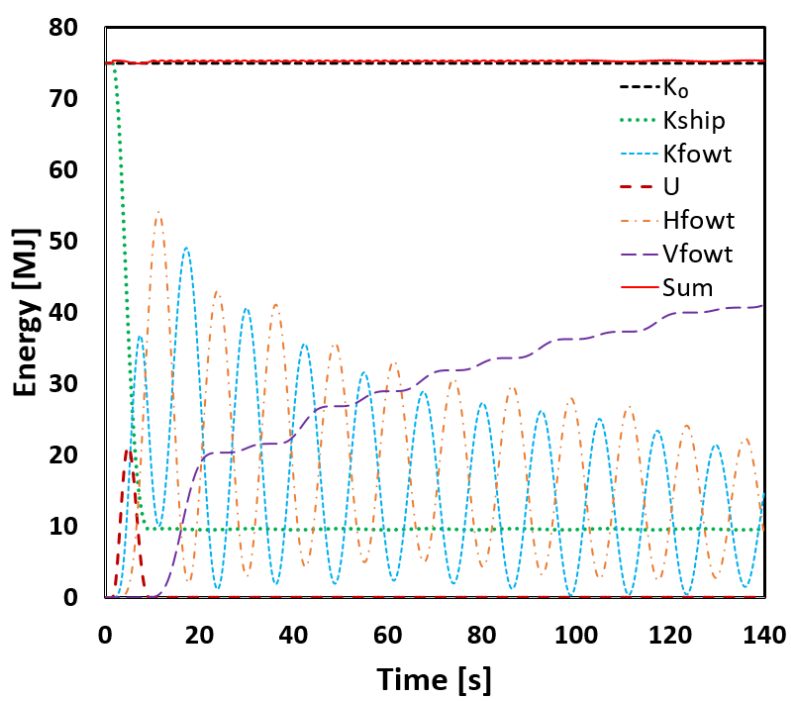

Figure 9. Energy balance - Viscous damping applied - 140s.

In Figure 8, by removing wave and viscous damping forces the total energy remains equal to $K_{0}$, and the energy balance seems to be consistent. As expected, the FOWT continues to pitch indefinitely as there are no hydrodynamic damping forces acting on it. 
When only viscous damping is considered (Figure 9), the motion of the FOWT, as well as $K_{\text {fowt }}$ and $H_{\text {fowt }}$, gradually decay from the moment of impact. The behavior of the FOWT appears to be physical and the energy balance is consistent.

On the other hand, when only wave damping is active (Figure 10), the FOWT presents an unexpected behavior: its pitch movement increases up to about 100 seconds and then begins to decay. Moreover, the FOWT's kinetic energy $K_{\text {fowt }}$, as well as the works of buoyancy $H$ and wave $W$ forces increase unrealistically beyond the initial kinetic energy $K_{0}$. So even though the total energy remains constant, the FOWT does not present a physical behavior, indicating an issue with the wave damping effect.

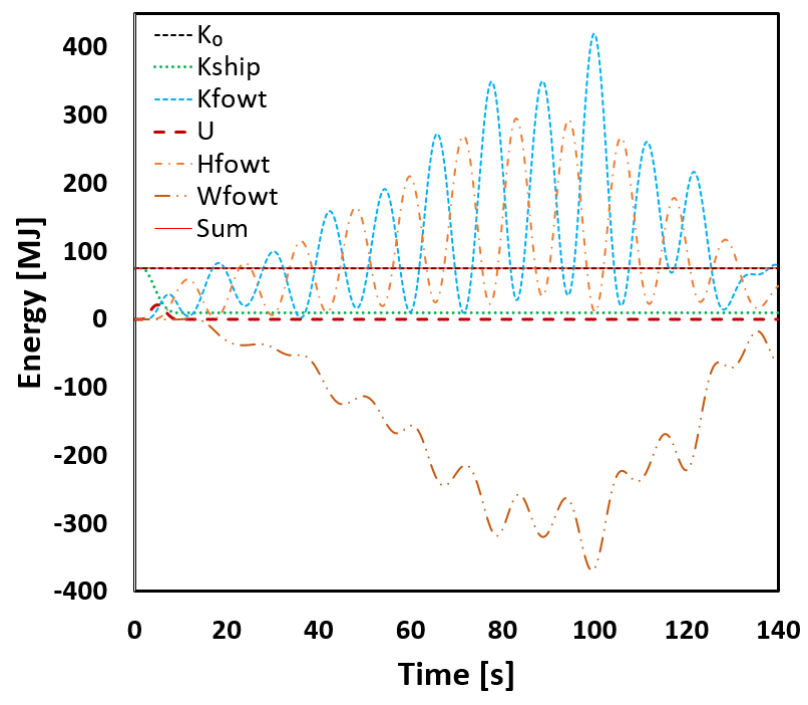

Figure 10. Energy balance - Wave damping applied - 140s.

In a nutshell, these simulations demonstrate that the gap occurring in the energy balance just after the ship's impact is due to the absence of the so-called crushed nodes kinetic energy, which is not included in the kinetic energy calculated by MCOL. Furthermore, while the viscous drag damping effect may be confidently considered in LS-Dyna/MCOL simulations, wave damping force calculations appear to be problematic, therefore this functionality should be used with caution.

\section{$3.3 \quad$ Model 3}

\subsubsection{Description}

To confirm the origin of the gap in the energy balance, let us now consider Model 3 presented in Figure 11.

In this model, the deformable semi-cylinder is now part of the FOWT and is connected to the rigid floater by a spring. As for the impactor, it is modelled with a rigid semi-cylinder. This new configuration aims to simulate in an isolated and controlled manner the deformation of the FOWT impacted by a rigid ship. By measuring the velocity $v_{\text {node }}$ of the single node that connects the deformable semi-cylinder to the spring element, and given the assembly's mass (1000 tons), it is possible to calculate the associated kinetic energy. This energy denoted $K_{\text {crushed }}$ is expected to be missing in the overall system energy balance postprocessed from the numerical results.

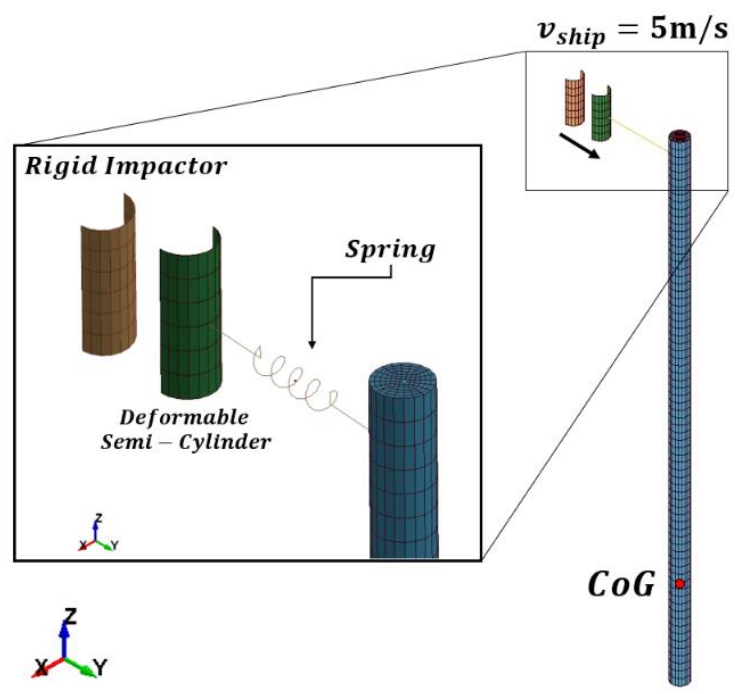

Figure 11. Model 3 setup.

\subsubsection{Results and Discussion}

Results for $v_{\text {node }}$ and $K_{\text {crushed }}$ over time are presented in Figure 12. After the impact, the measured node translates back and forth with the whole assembly and simultaneously vibrates. This behavior is reflected on both curves. The kinetic energy $K_{\text {crushed }}$ is added to the final energy balance, presented in Figure 13.

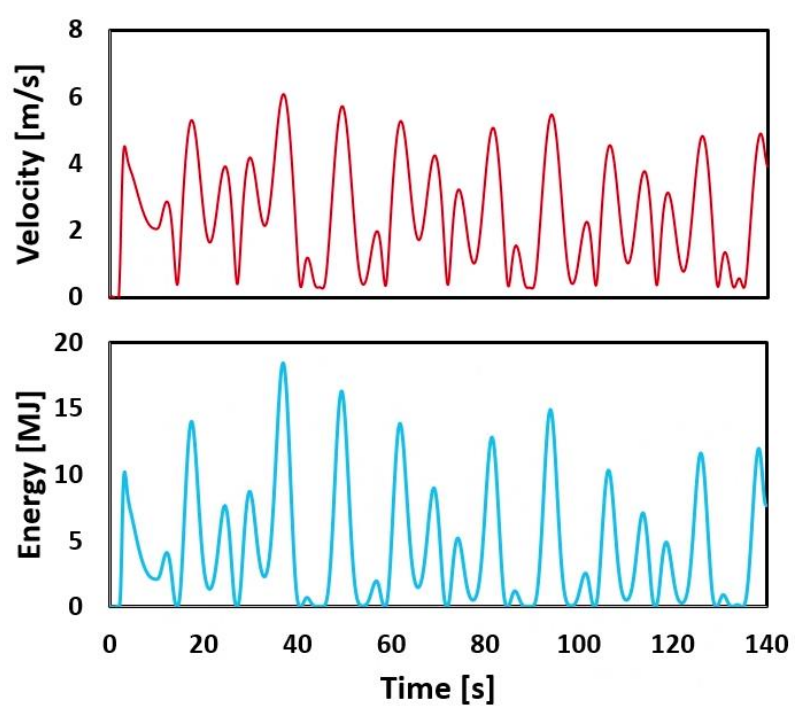

Figure 12 . Nodal velocity $\left(v_{\text {node }}\right)$ and calculated kinetic energy ( $\left.K_{\text {crushed }}\right)$ over time.

As expected, the total energy is equal to $K_{0}$. A comparison of the total energy obtained with and without $K_{\text {crushed }}$ is presented in Figure 14 clearly demonstrating the origin of the "missed energy" observed in Model 1. 


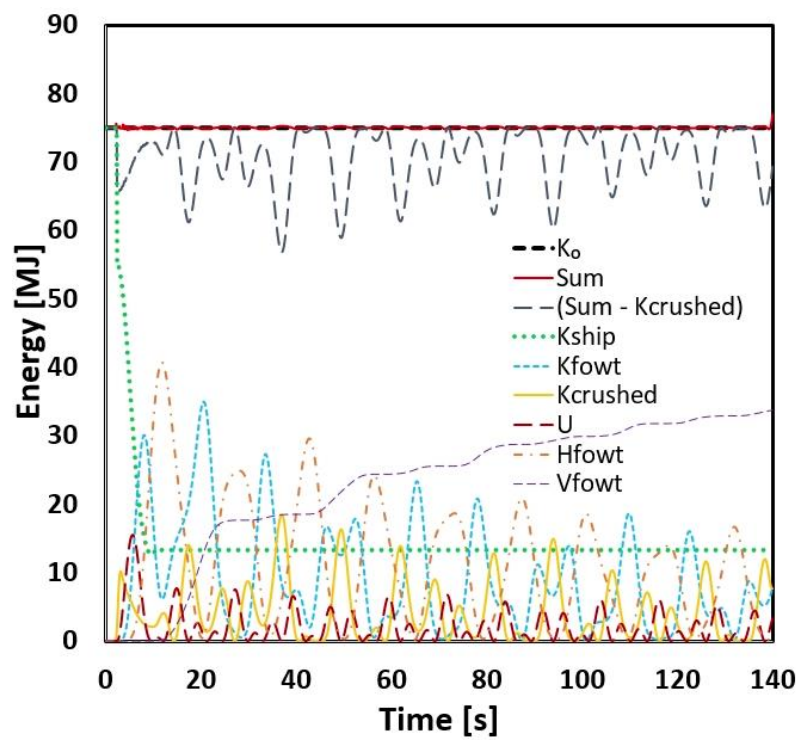

Figure 13. Energy balance, with and w/o "crushed nodes kinetic energy.

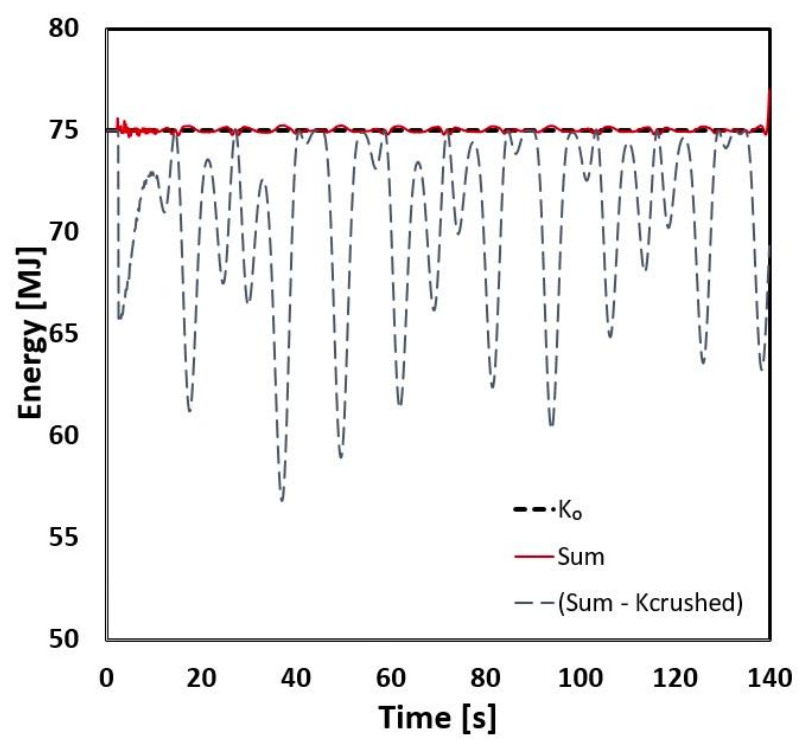

Figure 14. Comparison of the total energy with and without the calculated kinetic energy.

Finally, it is possible to confirm that the kinetic energy associated to the velocity of the nodes displaced in the deformation of the FOWT is significant. This component is, however, not calculated by MCOL, which explains the incomplete energy balance found previously.

\section{CONCLUSION}

Preliminary simulations of a ship-FOWT collision implemented with LS-Dyna's NLFE solver in conjunction with the MCOL subroutine have presented an incorrect energy balance: the total energy is not equal to the initial kinetic energy. In order to provide a detailed understanding of the involved mechanisms and enable a solid comprehension of the issue and its origin, three simplified ship-FOWT collision models have been developed.

In the first model, a rigid ship bow is impacting a deformable SPAR-like FOWT, inducing a large pitch movement on the latter. Results obtained from this model provide a good understanding of the impact mechanism in terms of energy dissipation and, most importantly, clearly show the gap between the total and the initial kinetic energy.

In the further simplified second model, a fully rigid SPAR-like FOWT is struck by an adapted impactor: a semi-cylinder connected via a spring to a rigid plate representing the colliding ship. The motion of the FOWT, limited to pitch rotation around its $\mathrm{CoG}$, is governed by MCOL, while the ship's movement is controlled by LS-Dyna. During and just after the impact, the energy balance is consistent, showing that the energy gap observed in the first model probably corresponds to the kinetic energy of the FOWT's crushed nodes. Moreover, by extending the simulation time, it is demonstrated that the wave radiation damping forces are not correctly handled in MCOL and this issue leads to an unrealistic pitch motion of the FOWT.

In the third simplified model, a rigid semi-cylinder representing the striking ship collides with a deformable one connected by a spring to the SPAR-like FOWT rigid cylinder. In this way, the kinetic energy of the FOWT's crushed part can be easily controlled, confirming that the gap observed in the first model indeed corresponds to this kinetic energy component, which is not calculated by MCOL.

The following conclusions are finally drawn:

-As the kinetic energy associated to the relative velocity (with respect to the $\mathrm{CoG}$ of the structure) of the deformed nodes is not accounted for by MCOL, the energy balance will always present a gap between the total energy and the initial kinetic energy.

-Although wave radiation damping forces seem to have a small effect in short-term collision analysis, the underlying theory is not implemented correctly in the MCOL subroutine and therefore cannot be used for long-term analysis.

\section{ACKNOWLEDGMENT}

This work was carried out within the framework of the WEAMEC, West Atlantic Marine Energy Community, and with funding from ICAM Engineering School, Pays de la Loire Region and Europe (European Regional Development Fund). 


\section{REFERENCES}

Brown, A. J. 2002. Collision scenarios and probabilistic collision damage. Marine Structures, 15(4-5), 335364.

Echeverry, S., Márquez, L., Rigo, P., \& Le Sourne, H. 2020. Numerical crashworthiness analysis of a spar floating offshore wind turbine impacted by a ship. Developments in the Collision and Grounding of Ships and Offshore Structures - Proceedings of the 8th International Conference on Collision and Grounding of Ships and Offshore Structures, ICCGS 2019, 004(2004), 85-95.

Le Sourne, H. 2007. A ship collision analysis program based on super-element method coupled with large rotational ship movement analysis tool. International Conference on Collision and Grounding of Ships, 131-138.

Le Sourne, H., County, N., Besnier, F., Kammerer, C., \& Legavre, H. 2003. LS-DYNA Applications in Shipbuilding. 4th European LS-DYNA Users Conference, 0, 1-16.

Le Sourne, H., Donner, R., Besnier, F., \& Ferry, M. 2001. External Dynamics of Ship-Submarine Collision. 2nd International Conference on Collision and Grounding of Ships (ICGS), 137-144.

Lee, S.-G., Nam, J.-H., Kim, J.-K., Zhao, T., \& Nguyen, H.-A. 2012. Structural safety assessment of ship collision using FSI analysis technique. TheTwentySecond International Offshoreand Polar Engineering Conference.

Minorsky, V. U. 1958. An analysis of ship collisions with reference to protection of nuclear power plants.

Paboeuf, S., Le Sourne, H., Brochard, K., \& Besnard, N. 2015. A damage assessment tool in ship collisions. Proceedings of Damaged Ship III-The Royal Institution of Naval Architects, 25-26.

Rudan, S., Ćatipović, I., Berg, R., Völkner, S., \& Prebeg, P. 2019. Numerical study on the consequences of different ship collision modelling techniques. Ships and Offshore Structures, 14(sup1), 387-400.

Song, M., Ma, J., \& Huang, Y. 2017. Fluid-structure interaction analysis of ship-ship collisions. Marine Structures, 55, 121-136.

Tabri, K. 2012. Influence of coupling in the prediction of ship collision damage. Ships and Offshore Structures, 7(1), 47-54.

Yu, Z., \& Amdahl, J. 2016. Full six degrees of freedom coupled dynamic simulation of ship collision and grounding accidents. Marine Structures, 47, 1-22.

Yu, Z., Amdahl, J., \& Storheim, M. 2016. A new approach for coupling external dynamics and internal mechanics in ship collisions. Marine Structures, 45, 110-132.

Yu, Z., Shen, Y., Amdahl, J., \& Greco, M. 2016. Implementation of linear potential-flow theory in the 6DOF coupled simulation of ship collision and grounding accidents. Journal of Ship Research, 60(3), 119-144. 